This document is confidential and is proprietary to the American Chemical Society and its authors. Do not copy or disclose without written permission. If you have received this item in error, notify the sender and delete all copies.

\title{
Bile Acid Profiling and Quantification in Biofluids using Ultra-Performance Liquid Chromatography Tandem Mass Spectrometry
}

\begin{tabular}{|r|l|}
\hline Journal: & Analytical Chemistry \\
\hline Manuscript ID & ac-2015-015564.R3 \\
\hline Manuscript Type: & Article \\
\hline Date Submitted by the Author: & n/a \\
\hline Complete List of Authors: & $\begin{array}{l}\text { Sarafian, Magali; Imperial College of London, Surgery and Cancer } \\
\text { Lewis, Matthew; Imperial College of London, Surgery and Cancer } \\
\text { Pechlivanis, Alexandros; Imperial College of London, Surgery and Cancer } \\
\text { Ralphs, Simon; Imperial College of London, Hepatology } \\
\text { Mc Phail, Mark; Imperial College of London, Hepatology } \\
\text { Patel, Vishal; Institute of Liver Sciences, King's College Hospital, Division of } \\
\text { Transplantation Immunology \& Mucosal Biology } \\
\text { Dumas, Marc-Emmanuel; Imperial College of London, Surgery and Cancer } \\
\text { Holmes, Elaine; Imperial College, Surgery and Cancer } \\
\text { Nicholson, Jeremy; Imperial College of London, Surgery and Cancer }\end{array}$ \\
\hline \hline
\end{tabular}

SCHOLARONE ${ }^{\text {m }}$

Manuscripts 


\title{
Bile Acid Profiling and Quantification in Biofluids using Ultra- Performance Liquid Chromatography Tandem Mass Spec- trometry
}

\author{
Magali H. Sarafian ${ }^{\dagger *}$, Matthew R. Lewis ${ }^{\dagger \text { †े }^{*}}$, Alexandros Pechlivanis $\dagger$, Simon Ralphs ${ }^{\S}$, Mark JW \\ McPhail $^{\S}$, Vishal C. Patel ${ }^{\#}$, Marc-Emmanuel Dumas ${ }^{\dagger}$, Elaine Holmes ${ }^{\dagger}$, Jeremy K. Nicholson ${ }^{\dagger * *}$ \\ $\dagger$ Imperial College of London, Division of Computational Systems Medicine, Department of Surgery and Cancer, Sir Alex- \\ ander Building, Exhibition Road, South Kensington, London SW7 2AZ, UK, \$ Imperial College of London, MRC NIHR \\ National Phenome Centre, Department of Surgery and Cancer, IRDB building, Du Cane Road, London W12 0NN, UK, \\ Imperial College London, Department of Hepatology, St Mary's Hospital, Paddington, London, \# King's College London, \\ Institute of Liver Sciences, Hospital NHS Foundation Trust, Division of Transplantation Immunology \& Mucosal Biology, \\ MRC Centre for Transplantation \\ * These authors contributed equally to the work
}

ABSTRACT: Bile acids are important end products of cholesterol metabolism. While they have been identified as key factors in lipid emulsification and absorption due to their detergent properties, bile acids have also been shown to act as signaling molecules and intermediates between the host and the gut microbiota. To further the investigation of bile acid functions in humans, an advanced platform for high throughput analysis is essential. Herein we describe the development and application of a 15 minute UPLC procedure for the separation of bile acid species from human biofluid samples requiring minimal sample preparation. High resolution time of flight mass spectrometry was applied for profiling applications, elucidating rich bile acid profiles in both normal and disease state plasma. In parallel, a second mode of detection was developed utilizing tandem mass spectrometry for sensitive and quantitative targeted analysis of 145 bile acid (BA) species including primary, secondary and tertiary bile acids. The latter system was validated by testing the linearity (LLOQ $0.25-10 \mathrm{nM}$ and ULOQ $2.5-5 \mu \mathrm{M})$, precision $(\approx 6.5 \%)$ and accuracy $(81.2-$ $118.9 \%$ ) on inter- and intra-day analysis achieving good recovery of bile acids (serum/plasma $88 \%$ and urine $93 \%$ ). The UPLCMS/MS targeted method was successfully applied to plasma, serum, and urine samples in order to compare the bile acid pool compositional difference between pre-prandial and post-prandial states, demonstrating the utility of such analysis on human biofluids.

Bile acids (BA) are major components of bile, synthesized from cholesterol in the hepatocytes of the liver and play fundamental roles in many physiological processes. BAs are well known as powerful emulsifiers of dietary lipids in the intestine, ${ }^{1,2}$ antimicrobial agents, ${ }^{3,4}$ and signaling molecules regulating their own synthesis. BAs are modulated by gut microbiota $^{5-8}$ and perturbations of the circulating BAs pool have been shown to contribute to development of liver and intestinal diseases. $^{9,10}$

Numerous reports have shown the structural diversity of BAs from cholesterol catabolism in the liver to microbial transformations in the intestine. ${ }^{11,12}$ BAs traverse the boundary between endogenous metabolism and symbiotic gut bacterial metabolism, acting as a strong link between humans and their intestinal microbiota. The pool of BAs is comprised of primary BAs synthesized in the liver (cholic acid and chenodeoxycholic acid), secondary BAs produced mainly in the gut via modification of primary BAs, and tertiary BAs which are formed in both the liver and gut via modification of secondary BAs, such as sulfation, glucuronidation, glucosidation and $N$ acetylglucosaminidation. ${ }^{13}$ In the intestinal lumen, gut microbiota are free to modulate the hepatic output through various reactions, which include deconjugation and dehydroxylation at specific sites to form secondary BAs. All BAs are subject to a cycle of absorption, modification in the liver (further conjugation), and excretion back to the gastrointestinal tract in a pro-

Page 1 of 13 
cess known as enterohepatic circulation. BA anabolism and biotransformation is thus a complex iterative process yielding a wide range of molecular variants. The abundance of these BA species also occupies a wide concentration range in humans with respect to biofluid and physiological / pathological phenotype (0.1-2000 nM). Given the biological significance of this diverse pool of molecules, a reliable platform for specific and sensitive analytical measurement is therefore needed However, the chemical diversity of $\mathrm{BA},{ }^{14}$ the wide concentration range in abundance in biofluids, and the molecular complexity of the biofluids themselves all pose analytical challenges for sensitive and selective analysis of BAs. ${ }^{15-17}$

Within the past decade, LC-MS has been heavily utilized for the separation and detection of BAs in human and animal model biofluid samples. ${ }^{9,18-34}$ The most comprehensive of these methods have a relatively long analytical cycle (> 30 minutes), precluding rapid sample analysis..$^{21,25,28,30,34}$ On the other hand, the most rapid methods ( $<10$ minutes) have limited BA coverage. ${ }^{19,33}$ The majority of separations have analytical times greater than 20 minutes and intermediate level of BA species coverage (ranging from 11 to 32 species monitored). ${ }^{9,18-34}$ Very few LC-MS methods report coverage of tertiary BAs such as sulfate conjugates which have recently been implicated in the important role of the gut microbiota in human metabolism. ${ }^{9,32,34-36}$

The analytical foundation of these methods is reversed-phase separation utilizing a $\mathrm{C}_{18}$ stationary phase, which is suitable for retention and separation of the diverse range of hydrophobicity present among BAs. ${ }^{37}$ Furthermore, all methods reviewed utilize methanol, acetonitrile, or a combination thereof as the strong eluent. Yet, the elution of ubiquitous blood matrix components such as phospholipids and triglycerides is known to benefit from the use of stronger solvents (e.g. 2propanol), increased column temperature, or both. ${ }^{38-40}$ The methods reviewed therefore risk the accumulation of large amounts of lipid content on the column and consequential carry-over of intact lipids and steady bleed of fatty acids during continuous sample analysis. Where present, these effects potentially compromise the BA measurement quality by competing for ionization in the electrospray process. These effects have been observed within our laboratory when using such methodology. ${ }^{20}$

To combat this, a desirable LC separation should be directly compatible with lipid-rich matrices such as blood and bile, allowing for the analysis of minimally processed samples without sacrificing the excellent retention and separation of BA species provided by a reversed-phase mechanism. The "dilute-and-shoot" method developed herein features chemical protein precipitation, removal of proteins by centrifugation, and direct analysis of the supernatant. This approach eliminates the potential for BA loss due to pre-processing steps such as SPE and the commonly applied procedure of sample drying prior to reconstitution with water, ${ }^{20}$ methanol, ${ }^{9,17}$ or a combination thereof. ${ }^{2,4,26,28,31,35}$ In this manner, selective enrichment or exclusion of $\mathrm{BA}$ species across a wide range of hydrophobicity is minimized or circumvented entirely.

The method described herein represents substantial development beyond the many methods currently available in the literature. Using UPLC-MS, it combines a high throughput analytical cycle (15 minutes) with simple and fast sample preparation, producing an analytical pipeline capable of supporting the rapid analysis of hundreds or thousands of samples. These goals are achieved without sacrificing the coverage of BA species, which is unprecedented in modern UPLC-MS applications. Of the $145 \mathrm{BA}$ species detected by targeted tandem mass spectrometry (MS/MS), 89 were tertiary sulfated BAs synthesized for the development of this assay. Fifty-seven of the 145 BAs were quantitatively measured using 16 commercially available stable labeled standards. Their measurements were validated according to established FDA criteria for accuracy and precision on intra/inter day, linearity, carry-over, stability and matrix effect. Recoveries were evaluated for 16 deuterated BAs spiked in three human biofluids; plasma, serum and urine. In addition, our BA method was validated by application to routine analysis of pre-prandial vs. post-prandial human plasma, serum and urine samples.

\section{EXPERIMENTAL SECTION}

Materials. Organic solvents (HPLC grade) used for the sulfation and precipitation and sodium sulfate were obtained from

Page 2 of 13 
Sigma Aldrich (Dorset, UK). All mobile phases were prepared with LC-MS grade solvents, formic acid and ammonium formate from Sigma Aldrich (Dorset, UK). Table S-1 shows the 73 BA standards including 36 non-conjugated, 12 conjugated with taurine, 9 conjugated with glycine and 16 deuterated internal standards obtained from Steraloids (Newport, RI) and Medical Isotopes (Pelham, USA). Table S-2 shows the 88 sulfated BA standards that were synthesized in house including 50 non-conjugated, 23 conjugated with taurine, 15 conjugated with glycine. Bile acid name abbreviations are provided at the conclusion of the manuscript, as well as in Tables S-1 and S2.

Collection of human plasma for bile acid profiling and targeted analysis. Blood plasma was collected into lithiumheparin for profiling analysis from patients with acute liver failure and cirrhosis as well as from healthy control subjects for use in the bile acid profiling application. All samples were centrifuged at $1,000 \mathrm{~g}$ at $4^{\circ} \mathrm{C}$ for $10 \mathrm{~min}$ and aliquoted and stored at $-80^{\circ} \mathrm{C}$. Ethical approval for the collection was obtained from the National Research Ethics Service Committee (Ref 12/LO/1417).

For targeted analysis, blood and urine samples were collected from healthy volunteers $(n=20)$ in either the pre- or postprandial state. Ten volunteers were fasted overnight and blood and urine samples were collected after $12 \mathrm{~h}$ (pre-prandial group). The remaining ten volunteers were given a high-fat meal and blood and urine samples were collected after $2 \mathrm{~h}$ (post-prandial group). Serum and EDTA-plasma were centrifuged at $1,000 \mathrm{~g}$ and $4^{\circ} \mathrm{C}$ for $10 \mathrm{~min}$ and aliquoted and stored at $-80^{\circ} \mathrm{C}$. This study was given ethical permission for conduct in the NHS by St Mary's Research Ethics Committee (Ref 09/H0712/82).

Sample preparation for profiling analysis of liver failure and cirrhosis patients. Samples were received on dry ice and stored at $-80^{\circ} \mathrm{C}$ until needed for preparation and analysis. All samples were thawed at $4^{\circ} \mathrm{C}$, transferred to $1 \mathrm{~mL}$ Eppendorf 96-deepwell plates (Eppendorf, Stevenage UK) and centrifuged at maximum speed in an Eppendorf $5810 \mathrm{R}$ equipped with an A-2-DWP-AT rotor (3486 $\mathrm{x} g$ ) for 15 minutes. Supernatant $(100 \mu \mathrm{L})$ was transferred to $0.5 \mathrm{~mL}$ Eppendorf 96- deepwell plates and $300 \mu \mathrm{L}$ of ice-cold methanol were added to each sample. ${ }^{41}$ All plates were heat sealed (Thermo Fisher Scientific, Hertfordshire UK), homogenized by vortexing for 15 min at $4^{\circ} \mathrm{C}$ using an Eppendorf MixMate (1400 RPM), and incubated at $-20^{\circ} \mathrm{C}$ for $20 \mathrm{~min}$. All samples were again centrifuged at $4^{\circ} \mathrm{C}(3486 \mathrm{xg})$ for 15 minutes prior to decanting of $200 \mu \mathrm{L}$ of supernatant to Eppendorf microplates for heat sealing and subsequent analysis.

Sample preparation for targeted analysis of human pre/post-prandial study. The same procedure described for the preparation of samples for profiling analysis was used for the targeted analysis samples, except the volume of sample and therefore ice-cold methanol $(1: 3 \mathrm{v} / \mathrm{v})$ was reduced to 50 $\mu \mathrm{L}$ and $150 \mu \mathrm{L}$ respectively. Additionally, $10 \mu \mathrm{L}$ of deuterated internal standards $(2.1 \mu \mathrm{M})$ in $\mathrm{H}_{2} \mathrm{O}$ /acetonitrile/2-propanol $(10: 6: 5, \mathrm{v}: \mathrm{v}: \mathrm{v})$ were added to the sample prior to the addition of methanol.

UPLC-MS profiling and MS/MS conditions. BA analysis was performed by ACQUITY ultra-performance liquidchromatography (UPLC) (Waters Ltd, Elstree, UK) coupled to either a Xevo G2-S Q-ToF mass spectrometer (for profiling application) or a Xevo TQ-S mass spectrometer (for targeted detection application) (Waters, Manchester, UK). In both cases, the MS system was equipped with an electrospray ionization source operating in negative ion mode (ESI).

The reversed-phase chromatographic method consisted of a mobile phase system, adapted from existing lipid profiling methods, ${ }^{38}$ paired with a shorter alkyl chain stationary phase (C8) to facilitate both the separation of BA species and the elution of lipidic matrix content. For this purpose, an ACQUITY BEH C8 column $(1.7 \mu \mathrm{m}, 100 \mathrm{~mm}$ x $2.1 \mathrm{~mm})$ was selected and used at an operating temperature of $60^{\circ} \mathrm{C}$. The mobile phase solvent A consisted of a volumetric preparation of $100 \mathrm{~mL}$ acetonitrile added to $1 \mathrm{~L}$ ultrapure water, with a final additive concentration of $1 \mathrm{mM}$ ammonium acetate and $\mathrm{pH}$ adjusted to 4.15 with acetic acid. Mobile phase solvent B consisted of a volumetric preparation of acetonitrile and 2propanol in a 1:1 mixture. The gradient separation is described in Table S-3 in supplemental information. Critically, the high organic wash step was adjusted in length for the complete

Page 3 of 13 
elution of observable phospholipids and triglycerides, precluding their accumulation on column. The injection volume of all samples was $5 \mu \mathrm{L}$. To minimize injector carry-over, 3 wash cycles of weak $\left(\mathrm{H}_{2} \mathrm{O}:\right.$ 2-propanol, 90:10) and strong (2propanol) solvent preparations were performed simultaneously with sample analysis.

Mass spectrometry parameters were as follows: capillary voltage was set at $1.5 \mathrm{kV}$, cone voltage at $60 \mathrm{~V}$, source temperature $150{ }^{\circ} \mathrm{C}$, desolvation temperature at $600{ }^{\circ} \mathrm{C}$, desolvation gas flow at $1000 \mathrm{~L} / \mathrm{h}$, cone gas flow at $150 \mathrm{~L} / \mathrm{h}$. Bile acid species yielding characteristic fragments when subjected to collision-induced dissociation were assayed using multiple reaction monitoring (MRM), while those that did not fragment were assayed by selected ion monitoring (SIM). The transitions for each of the BA standards and deuterated internal standards are provided in Table S-1 and Table S-2.

Optimization of bile acid sulfation. Lithocholic acid was selected for the optimization of the sulfation procedure as it is the most abundant BA in human where sulfation occurs on hydroxyl C3. ${ }^{42,43}$ Triplicate samples of lithocholic acid (LCA) (pure reference material) were prepared for sixteen sulfation reaction conditions. Sulfur trioxide-pyridine complex was resuspended in $\mathrm{CHCl}_{3}(5 \mathrm{mg} / \mathrm{mL})$ or pyridine and added to 20 $\mu \mathrm{L}$ of BA standards $(0.5 \mathrm{mg} / \mathrm{mL})$ with or without sodium sulfate. The reaction was stopped after $1 \mathrm{~h}$ or $24 \mathrm{~h}$ at $\mathrm{RT}$ or $55^{\circ} \mathrm{C}$ by evaporation to dryness. All samples were stored at $-80^{\circ} \mathrm{C}$. Prior to use in analysis, all samples were solubilized in $\mathrm{H}_{2} \mathrm{O}$ /acetonitrile/2-propanol (10:6:5, v:v:v).

Purification of sulfated bile acids. Following synthesis, the presence of sulfated BAs was confirmed by UPLC-MS/MS using the chromatographic method described above and multiple reaction monitoring for the $\left[\mathrm{HSO}_{4}\right]^{-} \mathrm{m} / \mathrm{z} 97$ moiety. Each of the 46 sulfated BAs was purified by individually tailored chromatographic separations of the reaction mixture using an Acquity UPLC equipped with an XBridge BEH C8 column $(3.5 \mu \mathrm{m}, 4.6 \mathrm{~mm} \times 150 \mathrm{~mm})$. The solvents used were the same as described above for the analytical method. The chromatographic separation of each sulfated BA was repeated a number of times and fractions were repeatedly collected using a Frac- tion Collector III (Waters, Manchester, UK) in order to amass sufficient material for subsequent experiments.

Optimization of MRM transitions and SIR. Source parameters such as collision energy and capillary voltage were optimized for each standard by direct infusion combined with the UPLC flow rate and appropriate solvents (e.g. UPLC-MS/MS conditions). Source parameters were optimized for glycine, taurine and sulfate fragmentations. These optimizations were automatically performed using Waters IntelliStart software (Waters) and then improved manually. Cone voltage was set to $60 \mathrm{~V}$ for all BAs as no significant variation in ionization during the optimization was observed between $10-60 \mathrm{~V}$.

Method validation. The BA targeted UPLC-MS/MS method was validated according to the bioanalytical guidance provided by the FDA. ${ }^{44}$ Linearity was evaluated for each BA over a concentration range of $0.05 \mathrm{nM}-5 \mu \mathrm{M}$. The limit of detection (LOD) was determined with a signal to noise $(\mathrm{S} / \mathrm{N})$ ratio $>3$, the lower limit of quantification (LLOQ) was determined with $\mathrm{S} / \mathrm{N}>5$ and $<20 \%$ of coefficient of variation (CV, standard deviation divided by the mean), and upper limit of quantification (ULOQ) was determined with an intensity level below the detector saturation which corresponds to the highest standard concentration in the calibration curve. Matrix effect assessment aimed to detect potential increase or suppression of BAs ionization due to presence of interfering analytes in samples.

Matrix effect was evaluated by comparing integrated peak area of deuterated standards spiked in solvent and spiked in plasma samples for the quality controls; QC1 (10 nM) and QC3 (100 $\mathrm{nM}$ ). Carry-over was tested by comparison of blanks vs QC1 and QC5 $(5 \mu \mathrm{M})$.

Percent error of accuracy and precision of the assay were assessed with three different concentrations of quality control (QC) prepared in solvent $\mathrm{H}_{2} \mathrm{O}$ /acetonitrile/2-propanol (10:6:5, v:v:v). The QC1 (10 nM), QC2 (50 nM) and QC3 (100 nM) and QC4 $(750 \mathrm{nM})$ precision and accuracy were validated on intraday (6 replicates analyzed on same day) and interday (one replicate analyzed on each of six different days).

$$
\begin{gathered}
\text { Percent error of accuracy }=\frac{\text { calculated concentration }}{\text { actual concentration }} \times 100 \\
\text { Precision }=\frac{\text { standard deviation }}{\text { mean }} \times 100
\end{gathered}
$$

Page 4 of 13 
Recoveries were evaluated for each deuterated internal standard on six replicates for each biofluid; plasma, serum and urine. Deuterated internal standards were spiked into samples before sample preparation (pre-spiked) and after sample preparation (post-spiked). Recoveries were calculated as follows; area under peaks of pre-spiked-sample divided by the area under peaks of post-spiked samples at QC1 and QC3.

MS data pre-processing. Waters raw data files were converted to NetCDF format and data were extracted via XCMS (v1.24.1) package with R (v2.11) software. MassLynx software 4.1 and Target Lynx 4.1 were used respectively for data acquisition and validation for this high throughput targeted method for quantification of BAs.

Multivariate Statistical Analysis. Principal components analysis (PCA) was carried out on the integrated BAs peaks with Pareto scaling using SIMCA P+ v13 (Umetrics, Umeå, Sweden). A standard univariate statistic, the Student's $t$-test, was carried out to establish significant variations in BA concentrations observed between the pre- vs. post-prandial volunteers.

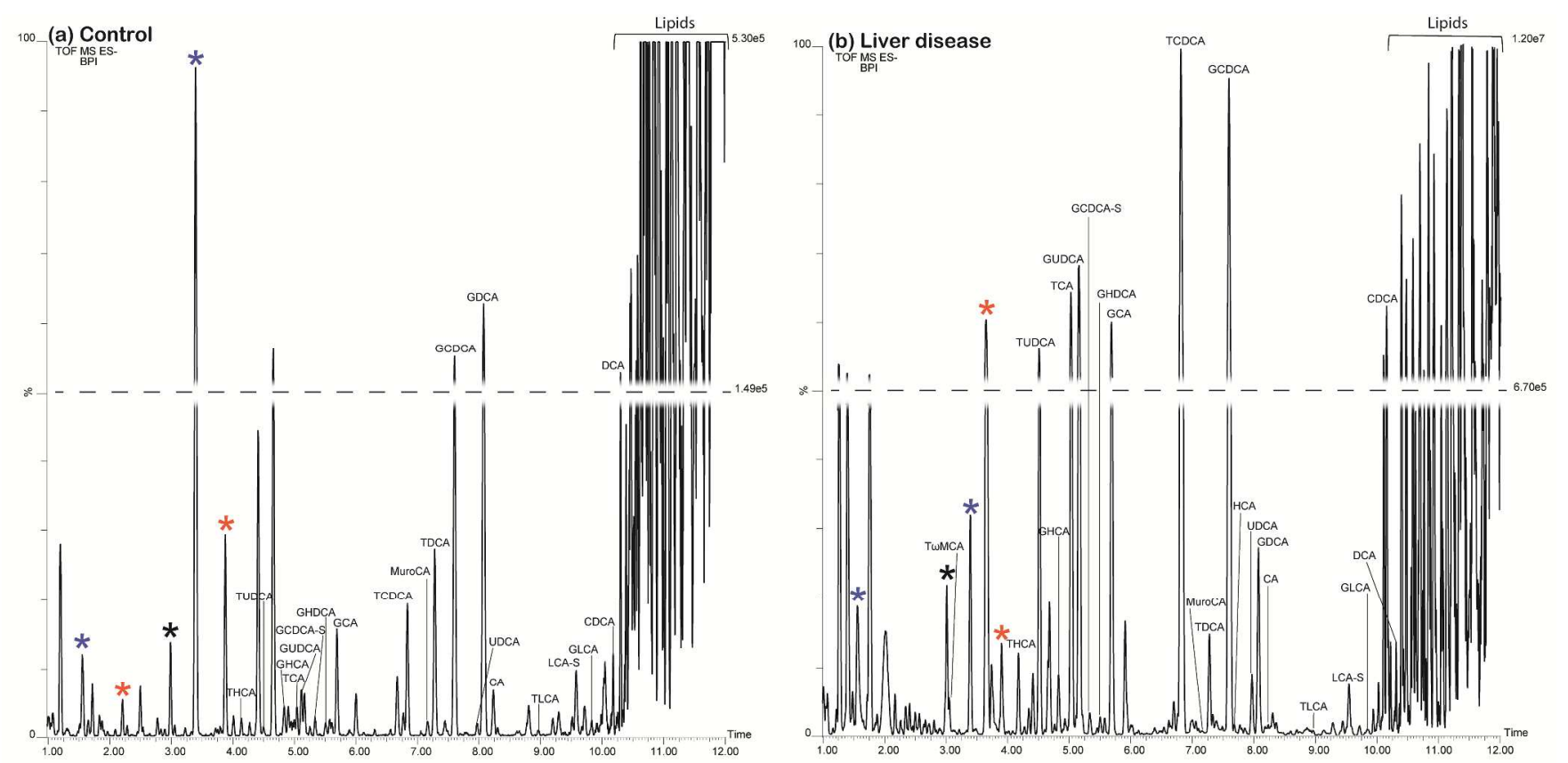

Figure 1. Bile acid profiling of human plasma from a single control sample (a) and pooled liver disease sample (b). Where possible, annotations of observed BAs were made from the 145 bile acid reference standards on hand. Furthermore, BAs with product ions such as glucuronides 113-175 m/z (*blue), sulfates $97 \mathrm{~m} / \mathrm{z}$ (*red) and glycine sulfates $74-97 \mathrm{~m} / \mathrm{z}$ (*black) were noted as such.

When paired with high resolution ToF MS detection, the sensitivity of the system was found to be sufficient for the detection of BA species in normal healthy plasma (Figure 1a). A

\section{RESULTS AND DISCUSSION}

Optimization of chromatographic conditions and profiling

application. A concentration of $1 \mathrm{mM}$ ammonium acetate was found to be sufficient for producing and maintaining adequate chromatographic peak shape across all BA species throughout the experiments presented in this study. Greater concentrations were observed to reduce the signal response from BA species, negatively impacting the sensitivity of the assay. Use of a low mobile phase $\mathrm{pH}$ was observed to substantially improve the retention of glycine conjugated and unconjugated BA species. The retention of taurine conjugated species was stable across the $\mathrm{pH}$ range achievable in reversed-phase separation $(\mathrm{pH} 3$ to 10). These data relating to mobile phase $\mathrm{pH}$ and $\mathrm{BA}$ retention are consistent with previously published results. ${ }^{24}$ The duration of the column washing step was determined by observation of phospholipid and triglyceride species elution (the latter only visible in positive mode ionization) during repeat injections of human plasma. generalized increase in serum BA species was observed in the profiling of a pooled plasma sample constructed from 191 subjects with acute liver failure and cirrhosis and used as an 
example here, demonstrating the utility of the profiling method for generating pathology specific signatures of bile acid dysregulation (Figure 1b). Although many of the bile acids observed in both the healthy control and disease pool sample analyses can be annotated from the 145 bile acids characterized in the targeted method below, additional signals relating to unidentified bile acids enhance the potential for deriving specific bile acid fingerprints for liver diseases and hence retain the capacity to contribute to understating the underlying mechanistic etiologies of these pathologies. In this example, the wide coverage profiling of primary, secondary and tertiary bile acids which allow differential mechanisms of intestinal and hepatic function to be measured on a single chromatogram could have significant translational potential for patients with acute liver failure, cirrhosis or cholestatic liver diseases.

Bile acid sulfation and purification. Sulfation of $46 \mathrm{BA}$ reference standards was conducted to generate sulfated reference materials for use in method development, facilitating a wide detection range of BA species. Sulfation efficiency was optimized by evaluation of sixteen reaction conditions selected from previous work. ${ }^{5,45,46}$

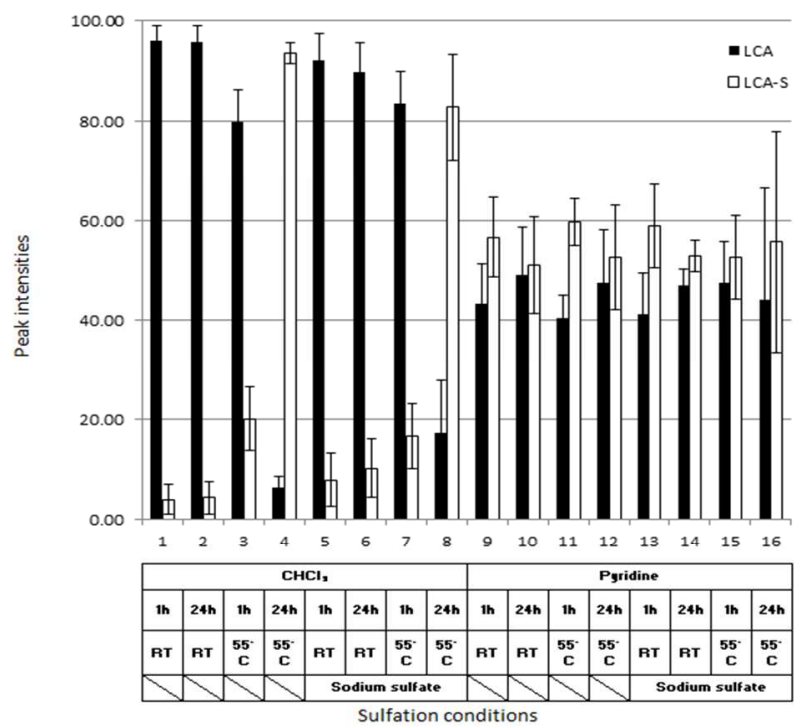

Figure 2. Optimization of BA sulfation. Sixteen conditions were tested on the lithocholic acid to evaluate the effect of time $(1 \mathrm{~h} v s$ $24 \mathrm{~h}$ ), temperature (RT room temperature $v s 55^{\circ} \mathrm{C}$ ), solvent (chloroform $\mathrm{CHCl}_{3}$ vs pyridine) and sodium sulfate. The error bars show the standard deviation of the replicates $(\mathrm{n}=3)$.

Different reaction conditions were tested such as the choice of solvent $\left(\mathrm{CHCl}_{3}\right.$ vs. Pyridine), reaction time (1h vs. $\left.24 \mathrm{~h}\right)$, reaction temperature $\left(22^{\circ} \mathrm{C} v s .55^{\circ} \mathrm{C}\right)$ and the presence or absence of sodium sulfate. Sulfation efficiency was assessed for sulfated lithocholic acid by integration of the area under the peak obtained by monitoring the $m / z$ transition $455.2472 \rightarrow 97$. The results of this procedural optimization are illustrated in Figure 2 and Table S-4. Analysis of sixteen conditions showed that the optimal sulfation efficiency was obtained with $\mathrm{CHCl}_{3}$, during 24 hours, at $55^{\circ} \mathrm{C}$ and with sodium sulfate (Figure S-1). Purification of the sulfated lithocholic acid was performed by HPLC separation with fraction collection. UPLC-MS/MS analysis was implemented to assess the purity of each fraction collected by characterization of two fragments; the sulfate moiety [HSO4] $]^{-} \mathrm{m} / \mathrm{z} 97$ and the non-sulfated form [M-H] (example $\mathrm{m} / \mathrm{z}$ for lithocholic acid 375.2904). This workflow was successfully applied for sulfation and purification of 46 BAs in total.

The C3 and C6 hydroxy groups are the major sites of naturally occurring BA sulfation in humans, while the $\mathrm{C} 3$ and $\mathrm{C} 7$ hydroxy sites are predominant in rodents. ${ }^{15,47}$ Naturally occurring sulfation on the $\mathrm{C} 12$ hydroxy group is still unclear in the literature. ${ }^{48}$ The in vitro reaction was capable of sulfation at each available hydroxy group, producing all applicable variants of mono-sulfated BAs for purification. The elution order observed in the reversed-phase HPLC separation of monosulfated BAs was used to determine the sulfate position, as previously reported $(\mathrm{C} 3<\mathrm{C} 6<\mathrm{C} 7<\mathrm{C} 12) .{ }^{49}$ Poly-sulfated BA products, however, were not observed. As previous studies have shown that liver sulfatase does not allow poly sulfation of BAs, their synthesis was not pursued. ${ }^{50}$

Targeted MS/MS analysis of $\mathbf{1 4 5}$ bile acids. In total, 145 BAs, including BA sulfates, were targeted in a single UPLCMS/MS method operating in a single mode of detection (Figure 3 and Table S-1 and S-2). The chromatographic method performed well in retaining all BA species, balancing the overall analysis time with high performance separation. However, the separation of unconjugated muricholic acid sterol isomers, differing only by the $\mathrm{OH}$ conformation in $\alpha, \beta, \omega$, was not achieved due to the early elution of the species. 
(a) Unconjugated bile acids

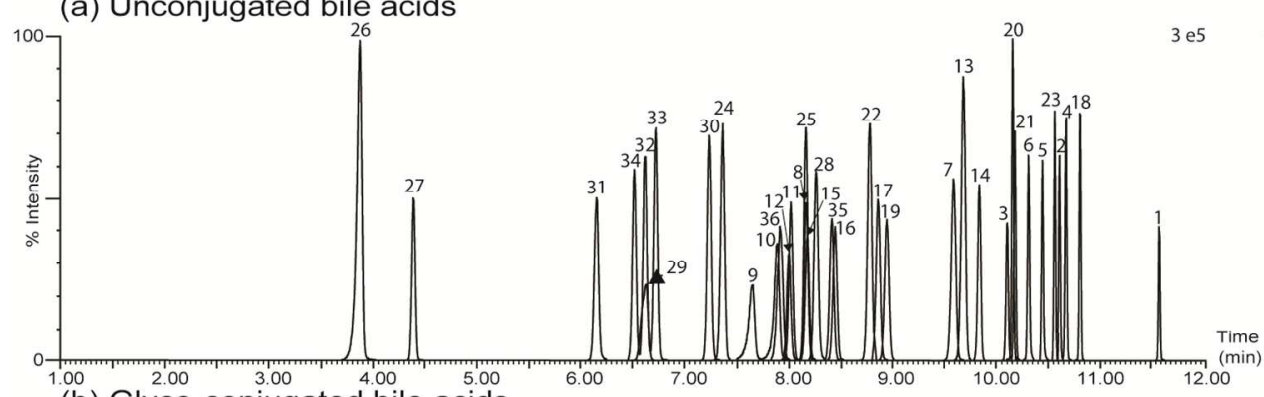

(d) Sulfated unconjugated bile acids

(b) Glyco-conjugated bile acids
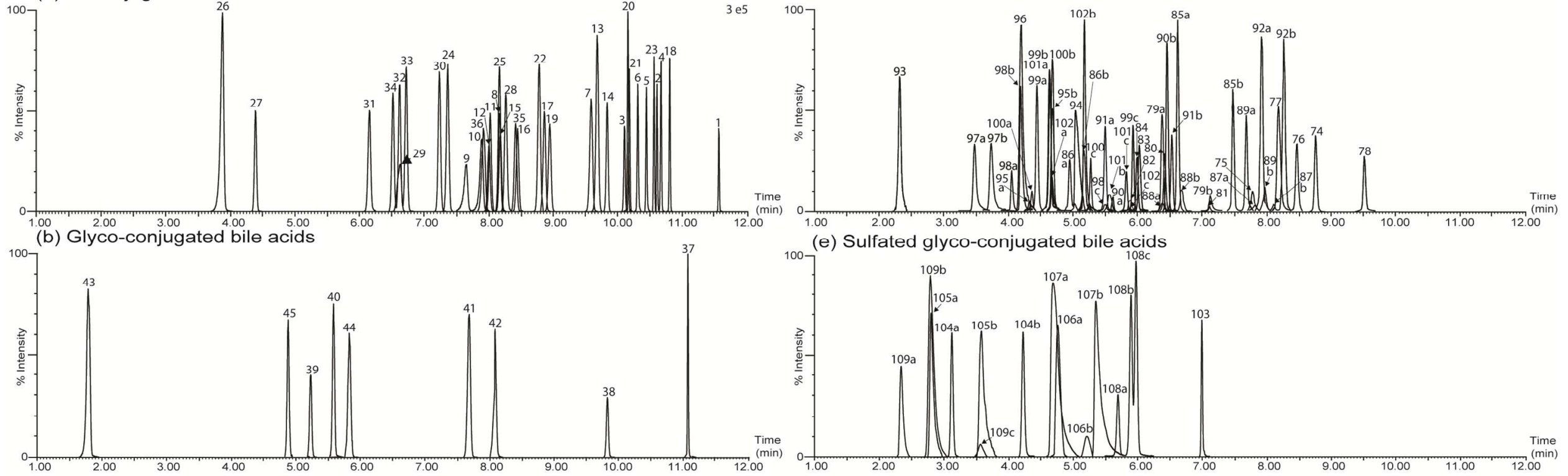

(e) Sulfated glyco-conjugated bile acids Timle
$(\mathrm{min})$
(c) Tauro-conjugated bile acids

-
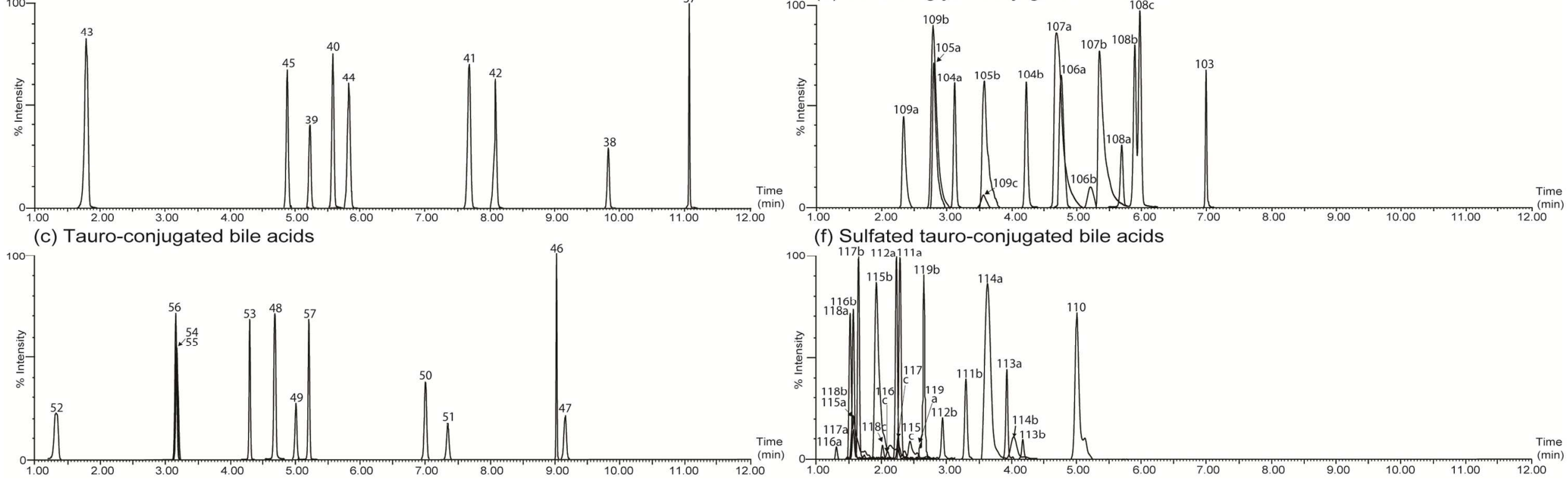

(f) Sulfated tauro-conjugated bile acids Time

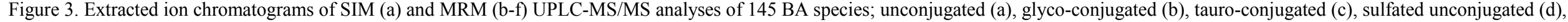
sulfated glyco-conjugated (e), sulfated tauro-conjugated (f). For more details see Table S-2 and S-3. 
As expected BA sulfates and also tauro- and glyco- conjugates eluted earlier than their unconjugated counterparts. This observation demonstrates that sulfated hydroxyls situated on the steroid nucleus change significantly the structure conformation and solubility of the molecule. These results are consistent with previous studies that demonstrate a change in physiological activity due to sulfation, especially for BA transport in the intestine. ${ }^{51}$ In addition to detection of sulfate conjugates, this chromatographic method offers the possibility to detect and quantify other water soluble BAs such as BA glucuronides. Furthermore, sulfation was implemented in this method as it is the most common modification observed in the BA elimination pathway and a simple one step procedure compared to glucuronidation which involve more complex organic synthesis steps. ${ }^{52}$

The combination of fit-for-purpose LC separation and optimized MRM transitions and SIM offered high sensitivity and selectivity for the $145 \mathrm{BA}$ species tested (Table S-1 and Table S-2). However, care needs to be taken for the mobile phase A of this chromatographic method which has to be accurately measured and stable if more solvents need to be added during the run (400 samples analyzed with $2.5 \mathrm{~L}$ of mobile phase A) as retention times of the glycine conjugated and unconjugated BA species are $\mathrm{pH}$ dependent. ${ }^{2}$ Therefore, this is an important consideration when targeting the MS detection time window to the elution of BA species, as any shift can result in missing the specific peaks.

\section{Method validation of the targeted UPLC-MS/MS method.} Optimization of MRM transitions and SIM aimed to maximize the detected signal for each characteristic ion species or transition. Afterwards, the UPLC-MS/MS method was validated in terms of accuracy and precision on intra/inter day, linearity, carry-over and matrix effect.

\section{Intra- and Inter day precision and accuracy.}

The LODs ranged between 0.05-7.5 $\mathrm{nM}$ and linearity was investigated over a wide concentration range of 10000 fold between $0.25 \mathrm{nM}$ and $5 \mu \mathrm{M}$ respectively LLOQ and ULOQ. This wide concentration range offered the best order of magnitude for quantification as the concentration of BAs spans a wide range of concentrations $(1 \mathrm{nM}$ to $3 \mu \mathrm{M}) .{ }^{27}$ Validation of the method shows high linear response associated with high mean $\mathrm{R}^{2}$ value of 0.998 . Overall, we obtained acceptable accuracy between $81.5-117.1 \%$ for intra-day and $81.2-118.9 \%$ for inter-day with acceptable precision between $1-15.5 \%$ for intraday and 2.1-19.9\% for inter-day analysis (Figure 4 and Table S-5). As can be seen, intraday precision and accuracy of the 57 BAs were consistent with inter-day precision and accuracy for the QC1, QC2, QC3 and QC4 compared to previous work that showed significant decreased of the inter-day accuracies. $^{27}$

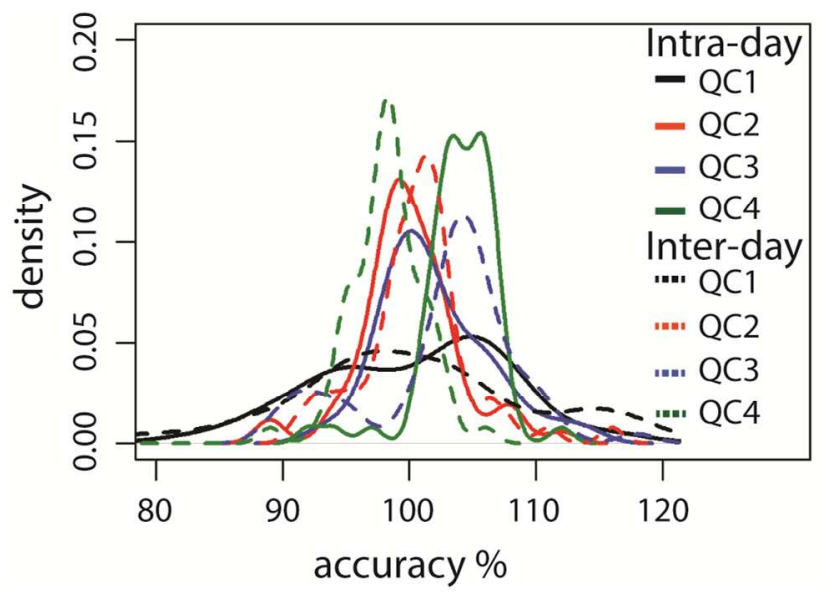

Figure 4. Accuracy distribution of the QC1 (10 nM), QC2 (50 $\mathrm{nM}), \mathrm{QC} 3(100 \mathrm{nM}), \mathrm{QC} 4(750 \mathrm{nM})$ on intra- and inter-day.

The density plots on Figure 4 summarize and compare intravs inter-day accuracies. The QC1 accuracy range was found to be 6\% higher than $\mathrm{QC} 2, \mathrm{QC} 3$ and QC4 accuracy range. In addition, the QC1 precision range was 8\% higher than QC2, QC3 and QC4 precision ranges. The QC1 was close to the noise, which might explain why it displayed a wider range of accuracy and precision values. Similar results have been found in previous work for QC1 where values were approaching the saturation limit. ${ }^{27}$ Conversely, QC2, QC3 and QC4, which benefit from being far from the noise and the saturation level, show the best accuracy and precision for intra/inter day analysis.

Bile acid recoveries in human serum, plasma and urine.

Prior to recovery evaluation, matrix effect and carry-over were measured. As can be seen in Table S-6 the ionization signal of deuterated standards in the presence of matrix $v s$ solvent were

Page 8 of 13 
between $87.05-111.85 \%$ for the QC1 and between 79.85 $111.37 \%$ for the QC3. Furthermore, qualitative measurement by combined infusion and injection of the standards confirmed that there was no significant ion suppression or enhancement. As shown in Table S-7 carry-over of BAs was easily removed by the UPLC system and is negligible. Extraction efficiency of the sample preparation was evaluated by analysis of recoveries and reproducibility $(\mathrm{n}=6)$ for each deuterated internal standard at their QC1 and QC3 concentrations (Figure S-2 and Table S8).

Simple protein precipitation is a straightforward one step sample preparation procedure that is known to reduce variation in lipid recoveries. ${ }^{41}$ High recoveries of BAs associated with high reproducibility were found in urine respectively around $92.7 \%$ and $8.58 \%$. Recovery of the standards in plasma and serum were around $4 \%$ less than for urine recoveries, whereas reproducibility around $2 \%$ higher than for urine. This difference between plasma/serum and urine can be explained by the presence of high concentrations of proteins in blood, which might affect the BA recoveries. Interestingly, ionization and recovery of bile acids coeluting with other lipid species after $10 \mathrm{~min}$ are not affected. Overall, satisfactory recoveries results were observed for all three biofluids.

\section{Application of the targeted UPLC-MS/MS methodology to} a human fed/fasted study showing post-prandial differences in bile acid quantification and detection. The validated targeted BA method was applied to a pre/post-prandial study. BA profiles of plasma, serum and urine human samples were investigated using a combination of univariate and multivariate analysis. As expected, the PCA scores plots for both plasma and serum showed clear separation of samples obtained pre- and post-prandially in PC1, reflecting systematic differences in the bile acid profile (Figure 5). Moreover, samples collected pre-prandially were more tightly clustered than those obtained following consumption of a fatty meal indicating that the dietary challenge may magnify inter-individual differences in BA synthesis. In total, around 66 BAs were detected in blood and 55 BAs in urine respectively including 15 and 22 sulfated BAs.
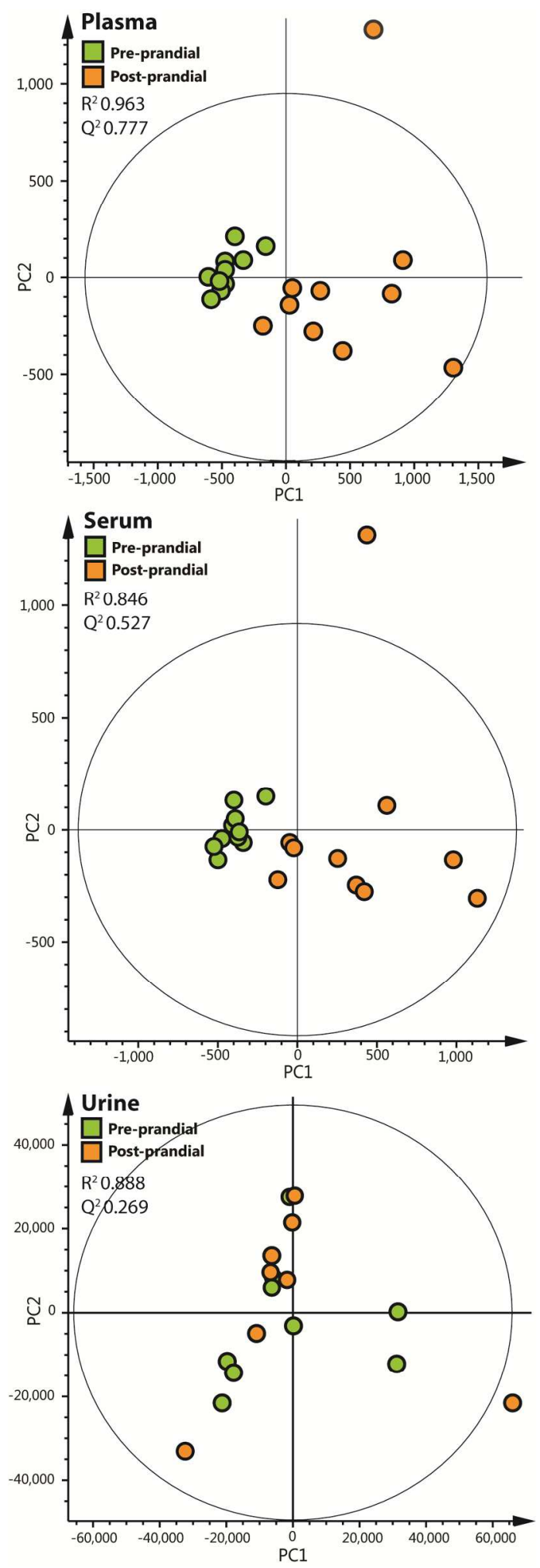

Figure 5. PCA scores plots of UPLC-MS/MS spectra obtained from pre/post-prandial human plasma, serum and urine.

In contrast, no obvious separation was observed between the pre- and post-prandial groups for the urine samples. A likely explanation for this observation would be that the urine was

Page 9 of 13 
collected too early, only 2 hours after the meal, which might be not long enough for the BAs to be accumulated in the urine.

Since the majority of BA excretion is via the fecal route, the urine should only contain minor concentrations of bile acids, and this may also account for the lack of observed variation between the pre- and post-prandial samples. For all three PCA models, the same outlier was observed but no modification in separation occurred on removal of this sample from any model.

The top five most significant BAs in discriminating between the pre- and post- prandial groups are listed in Table 1 and were consistent between serum and plasma. However, compared to a previous study, the order of significance was slightly different for the two matrices. ${ }^{19}$ Higher BA concentrations were observed in serum compared with EDTA-plasma, consistent with general observations in small molecule studies regarding the use of EDTA as an anticoagulant. ${ }^{53}$ We noted that GCDCA was the most concentrated BA in blood, yet its measurement remained within the dynamic range of the assay. The values observed in the present study are in agreement with those reported by Sherer and colleagues $(1710 \mathrm{nM}){ }^{19}$ but differ from those reported by other investigators (450-750 $\mathrm{nM}) .^{18,22,27,28}$ Subsequent application of the method described here to the analysis of independently obtained blood product samples have yielded results more closely aligned with the latter range, suggesting that the differences observed accurately reflect biological variance.

Table 1. BAs found in plasma and serum that discriminated between pre-prandial $v s$. post-prandial healthy volunteers with means and standard deviations of each quantified BAs. Statistical analysis was performed using 2-tailed student t-test.

\begin{tabular}{|c|c|c|c|}
\hline \multirow{2}{*}{$\begin{array}{l}\text { Biomarkers } \\
\text { Plasma }\end{array}$} & \multirow{2}{*}{ p-value } & \multicolumn{2}{|c|}{ Concentration (nM) } \\
\hline & & Pre-prandial & Post-prandial \\
\hline GCDCA & 2.11E-05 & $3300 \pm 1530$ & $13900 \pm 4540$ \\
\hline GCA & $8.00 \mathrm{E}-04$ & $23.31 \pm 7.45$ & $88.47 \pm 42.16$ \\
\hline GDCA & $1.70 \mathrm{E}-03$ & $10.34 \pm 50.32$ & $111 \pm 95.71$ \\
\hline TCA & $2.20 \mathrm{E}$ & $17.81 \pm 9.59$ & $51.63 \pm 25.55$ \\
\hline GLCA & $2.80 \mathrm{E}-03$ & $12.71 \pm 4.8$ & $27.24 \pm 11.32$ \\
\hline \multirow{2}{*}{$\begin{array}{c}\text { Biomarkers } \\
\text { Serum }\end{array}$} & \multirow{2}{*}{ p-value } & \multicolumn{2}{|c|}{ Concentration (nM) } \\
\hline & & Pre-prandial & Post-prandial \\
\hline GCDCA & $6.99 \mathrm{E}-05$ & $2200 \pm 1367$ & $11800 \pm 4614$ \\
\hline GDCA & $1.42 \mathrm{E}-03$ & $8.83 \pm 6.18$ & $108.47 \pm 69.66$ \\
\hline GCA & $2.73 \mathrm{E}-03$ & $17.41 \pm 6.87$ & $75.04 \pm 44.76$ \\
\hline GLCA & $3.76 \mathrm{E}-03$ & $5.36 \pm 2.48$ & $18.43 \pm 10.72$ \\
\hline TCA & $8.36 \mathrm{E}-03$ & $7.71 \pm 8.11$ & $28.37 \pm 19.11$ \\
\hline
\end{tabular}

\section{CONCLUSIONS}

Is it well established that BAs undergo multiple modifications during the enterohepatic circulation via interaction with the host or the gut microbiota. As a result, there is great BA diversity and most BAs found in human biofluidsare not listed in any database. The parallel development of a non-targeted screening approach for BAs combined with a targeted UPLCMS/MS BA assay provides a new resource for the profiling, identification and quantification of BAs found in human biofluids.

The method described is a reliable platform for BA analysis in human biofluids including those with lipidic matrices. Analysis of plasma, serum, and urine was efficiently performed with a high degree of precision in a rapid analytical cycle, and covering a large number of BA species. Of these species, 89 sulfated BAs have been described, enhancing the capability in the field for tertiary BA analysis. On the basis of these findings, this analytical method should provide new insights into the circulating BA pool regulated by the gut microbiota. Applications in biomarker discovery could furthermore provide guidance for clinical diagnosis and monitoring response to therapy in liver and intestinal diseases.

\section{ABBREVIATIONS}

BA, bile acid; UPLC-MS, ultra performance liquid chromatography-mass spectrometry; MS/MS, tandem mass spectrometry; SIM, selected ion monitoring; MRM, multiple reaction monitoring; CA, cholic acid; DCA; deoxycholic acid, HCA, hyocholic acid; LCA-S: lithocholic acid sulfate; MuroCA, murocholic acid; UDCA, ursodeoxycholic acid; GCDCA, glycochenodeoxycholic acid; GDCA, glycochenodeoxycholic acid sulfate; GDCA-S; glycodeoxycholic acid; GCA, glycocholic acid; GHCA, glycohyocholic acid; GHDCA, glycohyodeoxycholic acid; GLCA, glycolithocholic acid; GUDCA, glycursodeoxycholic acid; TCA, taurocholic acid; TDCA, taurodeoxycholic acid; TCDCA, taurochenodeoxycholic acid; THCA, taurohyocholic acid; THDCA, taurohyodeoxycholic acid; T $\omega$ MCA, tauro $\omega$ murichlic acid; TUDCA, tauroursodeoxycholic acid; S, sulfate; PCA, principal component analysis; QC, quality control; LOD, limit of detection; LLOQ, lower limit of quantification; ULOQ, upper limit of quantification.

\section{ASSOCIATED CONTENT}

\section{Supporting Information}

Additional information as noted in the text. This material is available free of charge via the Internet at http://pubs.acs.org. 


\section{AUTHOR INFORMATION}

\section{Corresponding Author}

**E-mail: j.nicholson@imperial.ac.uk (J.K.N.)

\section{ACKNOWLEDGMENT}

M.S. would like to thank Nestlé and M.Gaudin for his assistance for the sulfation optimization, as well as the staff of the MRCNIHR National Phenome Centre for their assistance and technical expertise.

MJWM is grateful to the Welcome Trust, UK for support during the production of this article. MJWM and VP would like to thank the staff of the Institute of Liver Studies and in particular Prof Julia Wendon and Dr Debbie Shawcross.

This work was supported by Nestlé ((RDLS015375), the Medical Research Council and National Institute for Health Research [grant number MC PC 12025] and by the Medical Research Council (MRC), reference [grant number MR/L001489/1].

\section{REFERENCES}

(1) Tso, P. Adv Lipid Res 1985, 21, 143-86.

(2) Cai, X.; Grant, D. J.; Wiedmann, T. S. J Pharm Sci 1997, 86, $372-7$

(3) Hofmann, A. F.; Eckmann, L. Proc Natl Acad Sci U S A 2006, 103, 4333-4.

(4) Inagaki, T.; Moschetta, A.; Lee, Y. K.; Peng, L.; Zhao, G.; Downes, M.; Yu, R. T.; Shelton, J. M.; Richardson, J. A.; Repa, J. J.; Mangelsdorf, D. J.; Kliewer, S. A. Proc Natl Acad Sci U S A 2006, 103, 3920-5.

(5) Anizan, S.; Bichon, E.; Di Nardo, D.; Monteau, F.; Cesbron, N.; Antignac, J. P.; Le Bizec, B. Talanta 2011, 86, 186-94.

(6) Chiang, J. Y. Am J Physiol Gastrointest Liver Physiol 2003, 284, G349-56.

(7) Claus, S. P.; Tsang, T. M.; Wang, Y.; Cloarec, O.; Skordi, E.; Martin, F. P.; Rezzi, S.; Ross, A.; Kochhar, S.; Holmes, E.; Nicholson, J. K. Mol Syst Biol 2008, 4, 219.

(8) Ridlon, J. M.; Kang, D. J.; Hylemon, P. B. J Lipid Res 2006, 47, 241-59.

(9) Duboc, H.; Rajca, S.; Rainteau, D.; Benarous, D.; Maubert, M. A.; Quervain, E.; Thomas, G.; Barbu, V.; Humbert, L.; Despras, G.; Bridonneau, C.; Dumetz, F.; Grill, J. P.; Masliah, J.; Beaugerie, L.; Cosnes, J.; Chazouilleres, O.; Poupon, R.; Wolf, C.; Mallet, J. M.; Langella, P.; Trugnan, G.; Sokol, H.; Seksik, P. Gut 2013, 62, 531-9.

(10) Jones, M. L.; Martoni, C. J.; Ganopolsky, J. G.; Labbe, A.; Prakash, S. Expert Opin Biol Ther 2014, 14, 467-82.

(11) Mahato, S. B.; Mukherjee, E.; Banerjee, S. Biotechnol Adv 1994, 12, 357-91.

(12) Russell, D. W. Annu Rev Biochem 2003, 72, 137-74.

(13) Marschall, H. U.; Matern, H.; Wietholtz, H.; Egestad, B.; Matern, S.; Sjovall, J. J Clin Invest 1992, 89, 1981-7.

(14) Moschetta, A.; Xu, F.; Hagey, L. R.; van Berge-Henegouwen, G. P.; van Erpecum, K. J.; Brouwers, J. F.; Cohen, J. C.; Bierman, M.; Hobbs, H. H.; Steinbach, J. H.; Hofmann, A. F. J Lipid Res 2005, 46, 2221-32.

(15) Griffiths, W. J.; Sjovall, J. J Lipid Res 2010, 51, 23-41.

(16) Street, J. M.; Trafford, D. J.; Makin, H. L. J Lipid Res 1983, 24, 491-511.

(17) Shimada, K.; Mitamura, K.; Higashi, T. J Chromatogr A 2001, 935, 141-72.

(18) Xie, G.; Wang, Y.; Wang, X.; Zhao, A.; Chen, T.; Ni, Y.; Wong, L.; Zhang, H.; Zhang, J.; Liu, C.; Liu, P.; Jia, W. J Proteome Res 2015, 14, 850-9.

(19) Scherer, M.; Gnewuch, C.; Schmitz, G.; Liebisch, G. J Chromatogr B Analyt Technol Biomed Life Sci 2009, 877, 3920-5.
(20) Want, E. J.; Coen, M.; Masson, P.; Keun, H. C.; Pearce, J. T.; Reily, M. D.; Robertson, D. G.; Rohde, C. M.; Holmes, E.; Lindon, J. C.; Plumb, R. S.; Nicholson, J. K. Anal Chem 2010, 82, 5282-9.

(21) Steiner, C.; von Eckardstein, A.; Rentsch, K. M. $J$ Chromatogr B Analyt Technol Biomed Life Sci 2010, 878, 2870-80.

(22) Perwaiz, S.; Tuchweber, B.; Mignault, D.; Gilat, T.; Yousef, I. M. J Lipid Res 2001, 42, 114-9.

(23) Sayin, S. I.; Wahlstrom, A.; Felin, J.; Jantti, S.; Marschall, H. U.; Bamberg, K.; Angelin, B.; Hyotylainen, T.; Oresic, M.; Backhed, F. Cell Metab 2013, 17, 225-35.

(24) Alnouti, Y.; Csanaky, I. L.; Klaassen, C. D. J Chromatogr B Analyt Technol Biomed Life Sci 2008, 873, 209-17.

(25) Bathena, S. P.; Mukherjee, S.; Olivera, M.; Alnouti, Y. $J$ Chromatogr B Analyt Technol Biomed Life Sci 2013, 942-943, 53-62.

(26) Huang, J.; Bathena, S. P.; Csanaky, I. L.; Alnouti, Y. J Pharm Biomed Anal 2011, 55, 1111-9.

(27) Garcia-Canaveras, J. C.; Donato, M. T.; Castell, J. V.; Lahoz, A. $J$ Lipid Res 2012, 53, 2231-41.

(28) Humbert, L.; Maubert, M. A.; Wolf, C.; Duboc, H.; Mahe, M.; Farabos, D.; Seksik, P.; Mallet, J. M.; Trugnan, G.; Masliah, J.; Rainteau, D. J Chromatogr B Analyt Technol Biomed Life Sci 2012, 899, 135-45.

(29) Sergi, M.; Montesano, C.; Napoletano, S.; Pizzoni, D.; Manetti, C.; Colistro, F.; Curini, R.; Compagnone, D. Chromatographia 2012, 75, 479-489.

(30) Qiao, X.; Ye, M.; Liu, C. F.; Yang, W. Z.; Miao, W. J.; Dong, J.; Guo, D. A. Steroids 2012, 77, 204-11.

(31) Ye, L.; Liu, S.; Wang, M.; Shao, Y.; Ding, M. J Chromatogr B Analyt Technol Biomed Life Sci 2007, 860, 10-7.

(32) Bobeldijk, I.; Hekman, M.; de Vries-van der Weij, J.; Coulier, L.; Ramaker, R.; Kleemann, R.; Kooistra, T.; Rubingh, C.; Freidig, A.; Verheij, E. J Chromatogr B Analyt Technol Biomed Life Sci 2008, $871,306-13$

(33) Chen, T.; Xie, G.; Wang, X.; Fan, J.; Qiu, Y.; Zheng, X.; Qi, X.; Cao, Y.; Su, M.; Xu, L. X.; Yen, Y.; Liu, P.; Jia, W. Mol Cell Proteomics 2011, 10, M110 004945.

(34) John, C.; Werner, P.; Worthmann, A.; Wegner, K.; Todter, K.; Scheja, L.; Rohn, S.; Heeren, J.; Fischer, M. J Chromatogr A 2014, 1371, 184-95.

(35) Swann, J. R.; Want, E. J.; Geier, F. M.; Spagou, K.; Wilson, I. D.; Sidaway, J. E.; Nicholson, J. K.; Holmes, E. Proc Natl Acad Sci U $S$ A 2011, 108 Suppl 1, 4523-30.

(36) Ridlon, J. M.; Kang, D. J.; Hylemon, P. B.; Bajaj, J. S. Curr Opin Gastroenterol 2014, 30, 332-8.

(37) Maekawa, M.; Shimada, M.; Iida, T.; Goto, J.; Mano, N. Steroids 2014, 80, 80-91.

(38) Shockor, J.; Crowe, H.; Yu, K.; Shion, H. Waters Application note 2011.

(39) Knittelfelder, O. L.; Weberhofer, B. P.; Eichmann, T. O.; Kohlwein, S. D.; Rechberger, G. N. J Chromatogr B Analyt Technol Biomed Life Sci 2014, 951-952, 119-28.

(40) Ogiso, H.; Suzuki, T.; Taguchi, R. Anal Biochem 2008, 375, 124-31.

(41) Sarafian, M. H.; Gaudin, M.; Lewis, M. R.; Martin, F. P.; Holmes, E.; Nicholson, J. K.; Dumas, M. E. Anal Chem 2014, 86, 5766-74.

(42) Norman, A.; Palmer, R. H. J Lab Clin Med 1964, 63, 9861001 .

(43) Palmer, R. H. Proc Natl Acad Sci U S A 1967, 58, 1047-50.

(44) Food and Drug Administration, FDA. US Department of Health and Human Services, center for Drug Evaluation and Research and center for Veterinary Medicine, Rockville, MD, 2013.

(45) Cerny, I.; Pouzar, V.; Budesinsky, M.; Bicikova, M.; Hill, M.; Hampl, R. Steroids 2004, 69, 161-71.

(46) Sandhoff, R.; Brugger, B.; Jeckel, D.; Lehmann, W. D.; Wieland, F. T. J Lipid Res 1999, 40, 126-32.

(47) Hagey, L. R.; Krasowski, M. D. Adv Nutr 2013, 4, 29-35. 
(48) Huijghebaert, S.; Parmentier, G.; Eyssen, H. J Steroid Biochem 1984, 20, 907-12.

(49) Alnouti, Y. Toxicol Sci 2009, 108, 225-46.

(50) Loof, L.; Hjerten, S. Biochim Biophys Acta 1980, 617, 192204.

(51) Palmer, R. H.; Bolt, M. G. J Lipid Res 1971, 12, 671-9.

(52) Mostarda, S.; Filipponi, P.; Sardella, R.; Venturoni, F.; Natalini, B.; Pellicciari, R.; Gioiello, A. Org Biomol Chem 2014, 12, 9592-600.

(53) Vuckovic, D. Anal Bioanal Chem 2012, 403, 1523-48. 
For Table of Contents Only

1

2

3

4

5

6

7

8

9

10

11

12

13

14

15

16

17

18

19

20

21

22

23

24

25

26

27

28

29

30

31

32

33

34

35

36

37

38

39

40

41

42

43

44

45

46

47

48

49

50

51

52

53

54

55

56

57

58

59

60

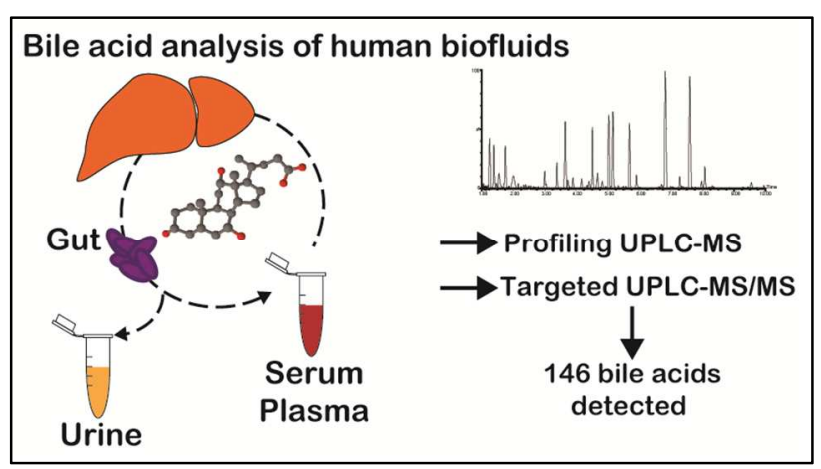

